Some of the literature published during, and subsequent to, the Second World War has been noted in the introduction: this cannot easily have been overcome; but a final chapter placing the latest studies in relation to their background would have been an advantage. While one agrees with the author's view that less speculation and more factual work on mycotrophy is required, one cannot help laying down the book with some disappointment that a connected account of this very important and widespread phenomenon has scarcely been achieved. Research workers will find much information within the covers; but it is scarcely a book to be recommended to students.

The format of the work maintains the high standard set by the publishers in their previous volumes; but it is suggested that reference to the figures and plates in the body of the text would be a decided advantage. Charles G. C. Chesters

\section{X-RAY STUDIES ON POLYMORPHISM}

\section{$X$-Ray Studies on Polymorphism}

Being a Collection of Papers concerning Submicroscopic Twinning in Crystals, with Appendices. By Prof. T. Ito, in collaboration with R. Sadanaga and Y. Takeuchi. Pp. vii+231. (Tokyo : Maruzen Co., Ltd., 1950.) 10 dollars.

$\mathrm{T}$ HIS book represents work done by Prof. T. Ito and his colleagues during the War period of isolation from scientific work in other countries. It starts off from the author's theory of polymorphism due to twinning, which covers also his concept of twinned space-groups. The author then presents new crystal-structure work on certain mineral groups in order to demonstrate the applicability of his theory. The groups studied comprise the following : eu- and epi-didymite, felspars, pyroxenes, amphiboles, epidote-zoisite, wollastonite and boléite; and there is also the organic substance TNT. This main part of the book covers some 130 pages.

The appendixes can be divided into two parts. The first part, of some fifty pages, contains new contributions to the determination of the crystal structures of the following minerals: tourmaline, kotoite, lièvrite (ilvaite), antigorite, ludlamite and orpiment. The first of these structures, in particular, has been under discussion for a long time, and there are still discrepancies between the structure put forward here and that published recently by Buerger and others.

The second part of the appendixes, some forty pages, is devoted to a full description of the new method of indexing powder photographs of an unknown substance in the general case (see Prof. Ito's letter in Nature, 164,$755 ; 1949$ ). It would be true to say that, up to the present, it has not been possible to index a powder photograph of an unknown substance where the symmetry of the specimen is lower than orthorhombic. The method put forward by the author overcomes the theoretical difficulty, even if troubles are met with in practice. 'The mathematical basis was laid by Runge in 1917, and in 1933 Delaunay published a method which enabled the smallest unit cell to be derived from arbitrarily chosen reciprocal. lattice vectors. This does not appear to have been followed up at the time. But in the present work the author gives a complete study of the method and also alternative methods devised by himself. The method demands considerable accuracy in the experi. mental data, and, as it depends on the entity of powder lines, it is very desirable that the errors should be uniformly distributed so far as possible. For this reason the author in his examples has used data obtained by means of focusing cameras. The general applicability and convenience in practice of this new method can only be tested over a number of years; but the author has now made it available for use by crystallographers. N. F. M. HENRY

\section{TEXT-BOOK OF OPTICS}

\section{Introduction to Theoretical and Experimental Optics}

By Prof. Joseph Valasek. Pp. $x+454 . \quad$ (New York : John Wiley and Sons, Inc.; London: Chapman and Hall, Ltd., 1949.) 52s, net.

HIS work is an attempt to provide, in one volume of ordinary text-book size, an introductory course in all branches of optics at a moderately advanced level. The idea is undoubtedly a sound one. The distinction between the various branches of optics as taught to undergraduates has been much too rigid in the past, and any attempt to bring them together is to be applauded.

The work is divided into four main parts. The first, headed "Geometrical Optics", is particularly valuable for its chapters on the physical limitations of optical images and on the brightness of images in instruments, both of which subjects are very often either neglected altogether, or treated mathematically at great length. Here, as in the whole of the book, a middle course is kept. The second section-"Physical Optics"-contains, besides the more usual material, discussions of the application of electromagnetic theory to reflexion from metallic media, refraction and dispersion. "Radiation and Spectra" is the heading of the third section, and it includes such topics as micro-wave absorption, magneto-optics and electro-optics, colour measurement and characteristic $\mathrm{X}$-ray emission, in addition to the chapters on thermal radiation and spectral theory. The fourth section is a laboratory manual for an experimental course which would normally be complementary to the three theoretical sections of the book.

It is inevitable that in a work of this scope some topics do not receive as full a treatment as they deserve, and Prof. Valasek himself points this out in his introduction. One cannot help feeling, however, that in some parts the number of topics mentioned might have been reduced with little loss, and the space used for fuller discussions of the more physical aspects of the results.

Large numbers of examples are provided at the end of each chapter, and a very useful bibliography to assist further study is included at the end. Throughout the text references to original papers are supplied, particularly with regard to recent work.

In addition to fulfilling its main purpose-the provision of a single text-book covering all branches of optics at honours-degree standard-this work will prove extremely valuable as a starting point for postgraduate students making a special study of some branch of optics or using optical techniques in some other field. 